\title{
Alteraciones del equilibrio acido base y electrolíticas en pacientes con crisis hiperglicémica atendidos en emergencia de un hospital general de Chiclayo
}

Electrolyte and acid-base abnormalities in patients with hyperglycemic crisis attended at emergency in a general hospital in Chiclayo

\author{
Robinsón León Zuloeta ${ }^{1, a}$, Gaudhy Sujhey Chávez Pasco ${ }^{2, b}$,Cesar Loza Munarriz ${ }^{3, c, d}$.
}

\section{RESUMEN}

Objetivo: Describir las alteraciones del equilibrio ácido base y electrolíticas en pacientes con crisis hiperglicémica atendidos en la emergencia de un hospital general de Chiclayo. Material y métodos: Serie de casos, prospectivo y de corte transversal; se seleccionaron pacientes diabéticos con crisis hiperglicémica que fueron vigilados por 3 horas. Se registraron los datos clínicos, demográficos, gases arteriales, glicemia y electrolitos. Resultados: Se evaluaron 52 pacientes con una edad promedio 55,1 $\pm 16,9$ y $29(55,8 \%)$ de sexo femenino. El tiempo promedio de diagnóstico fue 4,3 $\pm 5,4$ meses; 13 (25,0\%) eran episodios debut, 4 (7,7\%) fallecieron. El síntoma más frecuente fue confusión mental en $14(26,9 \%), 30(57,7 \%)$ tuvieron falla renal aguda. Las alteraciones electrolíticas más frecuentes fueron hiponatremia en $26(50,0 \%)$ e hipokalemia en $18(34,6 \%)$. La acidosis metabólica fue más frecuente en cetoacidosis que en coma hiperosmolar $(85,0 \mathrm{vs} 15,0 \% ; \mathrm{p}=0,000)$. La falla renal aguda ocurrió en $76,7 \%$ en cetoacidosis diabética y en $23,3 \%$ en el coma hiperosmolar $(p=0,74)$. Conclusiones: La frecuencia y las complicaciones de la cetoacidosis diabética y del coma hiperosmolar, así como de las alteraciones electrolíticas, muestran grandes diferencias con los datos reportados en la literatura.

PALABRAS CLAVE: Cetoacidosis diabética, coma hiperosmolar, trastornos hidroelectrolíticos, diabetes mellitus. (Fuente: DeCS BIREME).

\section{SUMMARY}

Objective: To describe electrolyte and acid-base imbalances in patients with hyperglycemic crisis attended at emergency in a general hospital in Chiclayo. Methods: A case series of diabetic patients with hyperglycemic crisis that were followed for 3 hours. Clinical, demographic, blood gases, serum glucose and electrolytes were gathered from patient's charts. Results: 52 patients were evaluated; mean age was $55.1 \pm 16.9$ years and $29(55.8 \%)$ were females. Mean time to diagnosis was $4.3 \pm 5.4$ months; 13 (25.0\%) occurred at onset of diabetes and $4(7.7 \%)$ died. The most common symptom was mental confusion in $14(26,9 \%)$ of patients, $30(57,7 \%)$ had acute renal failure.

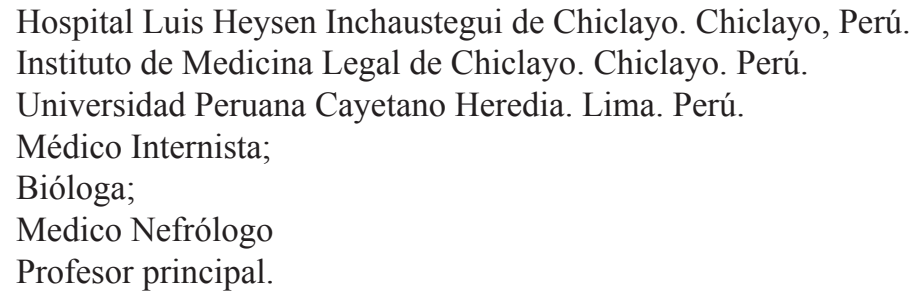


Hyponatremia in $26(50.0 \%)$ patients and hypokalemia in $18(34.6 \%)$ were the most common electrolyte abnormalities found. Metabolic acidosis was more frequently found in patients with ketoacidosis than in hyperosmolar coma (85.0 vs $15.0 \%$; $<0.0001$ ). Acute renal failure occurred in $76.7 \%$ among patients with ketoacidosis and in $23.3 \%$ of patients with hyperosmolar coma $(\mathrm{p}=0.74)$. Conclusions: the frequency and complications of diabetic ketoacidosis and of hyperosmolar coma as well as the electrolyte abnormalities differed from those reported in the literature.

KEYWORDS. Diabetic ketoacidosis, hyperosmolar coma, hydroelectrolytic disorders, diabetes mellitus. (Source: DeCS BIREME).

\section{INTRODUCCIÓN}

La Diabetes Mellitus (DM) es una patología de gran prevalencia con enorme impacto sobre la salud pública regional y mundial; investigaciones recientes concluyen que la prevalencia fluctúa entre 2 y $5 \%$ de la población mundial. En nuestro país, la prevalencia es variable según las regiones entre 1 a $8 \%$, siendo las ciudades con mayor prevalencia Lima y Piura (1).

La cetoacidosis diabética (CAD) y el estado hiperglicémico hiperosmolar (EHH), son las dos complicaciones metabólicas agudas más graves de la diabetes y los criterios diagnósticos de severidad están muy bien definidos por la Asociación Americana de Diabetes (ADA) (2). La crisis hiperglicémica (CHG), se presenta de manera súbita en pacientes diabéticos descompensados, con alteración del equilibrio ácido básico y de otros metabolitos que alteran el $\mathrm{pH}$ del organismo llevando a la acidosis metabólica. Las distintas formas de expresión de las CHG son la CAD y el EHH, no obstante, estos dos cuadros pueden presentarse de manera paralela y simultánea, lo que se denomina estado mixto (EM) (3).

Hay pocos estudios locales que aporten información sobre este problema en el Perú; un estudio reportó que el aspecto demográfico y epidemiológico es determinante en un tipo de pacientes (DM 2) hospitalizados por cetoacidosis diabética. En este estudio de 3683 pacientes, 3470 con DM 2, se encontraron 206 casos de cetoacidosis, $60,8 \%$ fueron diabéticos tipo 2, la media de edad fue 50,6 años y la permanencia hospitalaria 9,9 días. El factor precipitante más frecuente fue: infecciones en $42,2 \%$, patología cardiovascular $13,9 \%$ y en $30,6 \%$ ninguna de ellas; los autores concluyeron que la $\mathrm{CAD}$ en pacientes con $\mathrm{DM}$ 2 ha crecido sustancialmente y presenta una elevada tasa de mortalidad, principalmente en pacientes con mucha más edad (4).

En otro estudio, se evaluaron 59 pacientes con DM 2 y se encontró que $35(66 \%)$ pacientes ingresaron en estado grave con $\mathrm{pH}$ menor de $7 \mathrm{y}$ bicarbonato menor de 10; la glicemia promedio al ingreso fue $457 \pm 170$ $\mathrm{mg} / \mathrm{dl}, \mathrm{pH} 7,15 \pm 0,1$ y anión gap $24,45 \pm 7,44$. Los factores desencadenantes fueron la interrupción del tratamiento en $41 \%$, infección en $30 \%$, enfermedades intercurrentes (infarto miocárdico, accidente cerebrovascular) en $6 \%$, y ninguna causa aparente en $25 \%$ de los casos. Las complicaciones más frecuentes fueron síndrome hiperosmolar, CAD y causas mixtas (5).

Existen diferencias en la mortalidad entre los pacientes con CAD y EHH; la mortalidad por CAD es baja cercana a $2 \%$. Por lo tanto, las $\mathrm{CH}$ se constituyen en el factor más significativo de morbimortalidad en pacientes diabéticos. Los factores precipitantes más conocidos según la literatura, son las infecciones y la omisión en la administración de insulina (3).

En la región Lambayeque, hay diabéticos descompensados que hacen cuadros agudos de hiperglicemia; sin embargo, no existen datos estadísticos sobre ello, en varias revisiones bibliográficas de hospitales de Lima solo se describieron las complicaciones agudas de la diabetes, mientras que en estudios internacionales solo se reporta serie de casos, sobre frecuencia de trastornos acido base, $\mathrm{y}$ trastorno de la conciencia.

El objetivo del trabajo fue determinar las alteraciones hidroelectrolíticas y del equilibrio ácido base en pacientes con crisis hiperglicémica atendidos en emergencia de un hospital general docente de Chiclayo, así mismo, determinar la morbilidad y mortalidad.

\section{MATERIAL Y MÉTODOS}

Estudio tipo serie de casos prospectiva, de corte transversal, realizado en pacientes con crisis hiperglicémica, atendidos en emergencia del Hospital Regional Docente Las Mercedes de Chiclayo, de diciembre de 2014 a julio de 2015.

Los pacientes diabéticos diagnosticados con CAD y EHH, fueron seleccionados en el servicio de 
emergencia; el tipo de muestreo fue no probabilístico por conveniencia. Se excluyeron pacientes con falla renal crónica con una tasa de filtración glomerular (TFG) menor de $15 \mathrm{ml} / \mathrm{min}$.

Las variables de estudio fueron: complicaciones agudas de la diabetes mellitus 2, edad, sexo, tiempo de diagnóstico, alteraciones del sodio, alteraciones del potasio, alteraciones del equilibrio ácido base, injuria renal aguda, presencia de infección y mortalidad. Las complicaciones agudas de la diabetes mellitus 2, se definieron como cetoacidosis diabética y enfermedad hiperosmolar de acuerdo a los criterios de la ADA. Los criterios para cetoacidosis diabética fueron: $\mathrm{pH}$ $<7,3$, bicarbonato menor de $18 \mathrm{mmol} / \mathrm{l}$, osmolaridad sérica efectiva menor de $320 \mathrm{mOsm} / 1$ determinada por formula de dos veces el sodio más glucosa entre 18, presencia de cuerpos cetónicos en orina y sangre, y estado de conciencia alerta. Los criterios para enfermedad hiperosmolar fueron: $\mathrm{pH}>7,3$; bicarbonato mayor de $18 \mathrm{mmol} / \mathrm{l}$, osmolaridad sérica mayor de $320 \mathrm{mmos} / 1$, ausencia de cuerpos cetónicos en orina y sangre, y estado de conciencia confuso o coma (2). Las alteraciones del sodio y del potasio fueron categorizados según los valores estandarizados para hiponatremia (sodio $<135 \mathrm{mEq} / \mathrm{l}$ ), hipernatremia (sodio $>145 \mathrm{mEq} / \mathrm{l}$ ), hipokalemia (potasio menor de $3,5 \mathrm{mEq} / \mathrm{l}$ ) e hiperkalemia (potasio mayor de 5,5 $\mathrm{mEq} / \mathrm{l})$ y ausencia de alteraciones. Las alteraciones del equilibrio acido base fueron categorizados como acidosis metabólica en trastorno primario $(\mathrm{pH}<7,1)$, alcalosis respiratoria como trastorno secundario $\left(\mathrm{PCO}_{2}\right.$ $<30$ ), acidosis respiratoria o alcalosis metabólica o ausencia de alteraciones de la gasometría. La injuria renal aguda se midió por la tasa de filtración glomerular definida según clasificación AKIN(KDIGO) a toda variación de los niveles de creatinina por encima de 1,$5 ; 2$ y 3 veces su valor normal y descenso de la tasa de filtración glomerular, calculada por la ecuación de Cockcroft: 25,50 y $75 \%$ de su valor respectivamente.

Los datos fueron recolectados y registrados por el investigador, en una ficha de datos al momento del ingreso a la emergencia, y los exámenes bioquímicos procesados en el laboratorio del hospital. Se utilizó la estadística descriptiva, en las variables categóricas se

Tabla 1. Características clínicas y demográficas de los pacientes diabéticos descompensados que ingresaron a emergencia del hospital general de Chiclayo.

\begin{tabular}{lc}
\hline Características clínicas y demográficas & n (\%) \\
\hline Edad(años) & $55,2 \pm 16,9$ \\
Sexo & \\
Femenino & $29(55,8 \%)$ \\
Masculino & $23(44,2 \%)$ \\
IMC (kg/m²) & \\
Delgado & $4(7,7 \%)$ \\
Normal & $20(38,5 \%)$ \\
Obeso & $14(26,9 \%)$ \\
Sobrepeso & $14(26,9 \%)$ \\
Tiempo de diagnóstico (años) & $4,39 \pm 5,5$ \\
Debut de Diabetes & $13(25 \%)$ \\
Si & \\
Antecedente de hipertensión arterial & $8(15,4 \%)$ \\
Si & \\
Uso de Antidiabéticos Orales & $24(50 \%)$ \\
Si & \\
Uso de IECA & $4(7,7 \%)$ \\
Si & \\
Condición Final & $48(92,3 \%)$ \\
Vivo & $4(7,7 \%)$. \\
Fallecido &
\end{tabular}


determinaron frecuencia absoluta y relativa, y en las variables continuas, medias y desviación estándar, o rango inter cuartil, según el tipo de distribución. Para la inferencia estadística, en las variables categóricas se utilizó Chi cuadrado y Prueba exacta de Fisher, y para las variables continuas, t-test o Suma de Rangos de Wilcoxon, dependiendo del tipo de distribución. Los datos fueron analizados con el Programa Stata/MP v. 15.

Los pacientes y familiares fueron informados de su participación en el estudio; luego firmaron el consentimiento informado. La investigación fue revisada y aprobada por el Comité Institucional de Ética para humanos de la Universidad Peruana Cayetano Heredia, así como del Hospital Regional Docente Las Mercedes de Chiclayo.

\section{RESULTADOS}

Se incluyeron 52 pacientes; la edad promedio fue $55,2 \pm 16,9$, y $29(55,8 \%)$ fueron de sexo femenino (tabla 1).

Las características clínicas relevantes fueron: confusión $14(26,9 \%)$ pacientes, polaquiuria $5(9,6 \%)$ $\mathrm{y}$ tos $5(9,6 \%)$ (tabla 2$)$.

Las complicaciones relacionadas a la diabetes más frecuente fue cetoacidosis diabética en $39(75 \%)$ y el enfermedad hiperosmolar en $13(25 \%)$ de pacientes. En cetoacidosis diabética fallecieron tres $(7,7 \%)$ pacientes y en enfermedad hiperosmolar falleció uno $(7,7 \%)$ (tabla 3$)$.

Tabla 2. Características clínicas de ingreso a la emergencia de pacientes diabéticos descompensados en un hospital general de Chiclayo.

\begin{tabular}{lcc}
\hline Síntomas & $\mathbf{n}$ & $\mathbf{\%}$ \\
\hline Confusión & 14 & 26,9 \\
Diarrea & 4 & 7,7 \\
Polaquiuria & 5 & 9,6 \\
Tos & 5 & 9,6 \\
Disuria & 4 & 7,7 \\
dolor abdominal & 4 & 7,7 \\
Disnea & 3 & 5,8 \\
Expectoración & 3 & 5,8 \\
Cefalea & 3 & 5,8 \\
Polidipsia & 3 & 5,8 \\
Somnolencia & 3 & 5,8 \\
Baja de peso & 1 & 1,9 \\
\hline
\end{tabular}

Tabla 3. Complicaciones agudas de los pacientes diabéticos ingresados a emergencia de un hospital general docente de Chiclayo.

\begin{tabular}{lcc}
\hline Complicaciones & $\mathbf{n}$ & $\mathbf{\%}$ \\
\hline Injuria Renal Aguda & 30 & 57,7 \\
$\mathrm{Si}$ & 22 & 42,3 \\
$\mathrm{No}$ & & \\
Alteraciones del sodio & 26 & 50,0 \\
$\quad$ Hiponatremia & 13 & 25,0 \\
Hipernatemia & 13 & 25,0 \\
Sodio Normal & & \\
Alteraciones del potasio & 19 & 36,5 \\
Potasio Normal & 18 & 34,6 \\
Hipokalemia & 15 & 28,8 \\
Hiperkalemia &
\end{tabular}


Tabla 4. Características clínicas demográficas y bioquímicas de los pacientes diabéticos descompensados que ingresaron por emergencia de un hospital general docente de Chiclayo.

\begin{tabular}{|c|c|c|c|c|c|}
\hline \multirow{2}{*}{ VARIABLE CATEGÓRICA } & \multicolumn{2}{|c|}{ CETOACIDOSIS } & \multicolumn{2}{|c|}{$\begin{array}{c}\text { COMA } \\
\text { HIPEROSMOLAR }\end{array}$} & \multirow{2}{*}{$\mathbf{p}$} \\
\hline & $\mathbf{n}$ & $\%$ & $\mathbf{n}$ & $\%$ & \\
\hline \multicolumn{6}{|l|}{ Sexo } \\
\hline Masculino & 18 & 46,2 & 5 & 38,5 & 0,629 \\
\hline Femenino & 21 & 53,8 & 8 & 61,5 & \\
\hline \multicolumn{6}{|l|}{ Índice de masa corporal } \\
\hline Delgado & 2 & 5,1 & 2 & 15,4 & 0,253 \\
\hline Normal & 14 & 35,9 & 6 & 46,1 & \\
\hline Obeso & 10 & 25,6 & 4 & 30,8 & \\
\hline Sobrepeso & 13 & 33,3 & 1 & 7,7 & \\
\hline \multicolumn{6}{|l|}{ Debut } \\
\hline $\mathrm{Si}$ & 9 & 23,1 & 4 & 30,8 & 0,579 \\
\hline No & 30 & 76,9 & 9 & 69,2 & \\
\hline \multicolumn{6}{|l|}{ Ocupación } \\
\hline Desocupado & 22 & 56,4 & 9 & 69,2 & 0,415 \\
\hline Ocupado & 17 & 43,6 & 4 & 30,8 & \\
\hline \multicolumn{6}{|l|}{ Tratamiento } \\
\hline Anti-diabéticos & 18 & 46,1 & 6 & 46,1 & 0,984 \\
\hline Insulina & 3 & 7,7 & 1 & 7,7 & \\
\hline Ninguno & 18 & 46,1 & 6 & 46,1 & \\
\hline \multicolumn{6}{|l|}{ Trastorno ácido base primario } \\
\hline Acidosis metabólica & 34 & 100,0 & 6 & 46,1 & 0,000 \\
\hline Alcalosis respiratoria & 0 & 0,0 & 6 & 46,1 & \\
\hline Acidosis respiratoria & 0 & 0,0 & 0 & 0,0 & \\
\hline Alcalosis metabólica & 0 & 0,0 & 1 & 7,7 & \\
\hline \multicolumn{6}{|l|}{ Trastorno ácido base secundario } \\
\hline Alcalosis respiratoria & 13 & 61,9 & 5 & 62,5 & 0,038 \\
\hline Acidosis respiratoria & 7 & 33,3 & 0 & 0,0 & \\
\hline Acidosis metabólica & 1 & 4,8 & 1 & 12,5 & \\
\hline Alcalosis metabólica & 0 & 0,0 & 2 & 25,0 & \\
\hline \multicolumn{6}{|l|}{ Injuria renal Aguda $* *$} \\
\hline $\mathrm{Si}$ & 23 & 59,0 & 7 & 53,8 & 0,746 \\
\hline No & 16 & 41,0 & 6 & 46,2 & \\
\hline \multicolumn{6}{|l|}{ Alteración del sodio } \\
\hline Hipernatremia & 9 & $23,1 \%$ & 4 & $30,8 \%$ & 0,630 \\
\hline Hiponatremia & 21 & $53,8 \%$ & 5 & $38,4 \%$ & \\
\hline Normal & 9 & $23,1 \%$ & 4 & $30,8 \%$ & \\
\hline \multicolumn{6}{|l|}{ Alteración del potasio } \\
\hline Hiperkalemia & 12 & $30,8 \%$ & 3 & $23,1 \%$ & 0,319 \\
\hline Hipokalemia & 15 & $38,4 \%$ & 3 & $23,1 \%$ & \\
\hline Normal & 12 & $30,8 \%$ & 7 & $53,8 \%$ & \\
\hline \multicolumn{6}{|l|}{ Condición final } \\
\hline Fallecido & 3 & $7,7 \%$ & 1 & $7,7 \%$ & 1,000 \\
\hline Vivo & 36 & $92,3 \%$ & 12 & $92,3 \%$ & \\
\hline
\end{tabular}

*Variables categóricas

**Con criterios KDIGO 
Tabla 5. Características clínicas, demográficas y bioquímicas de los pacientes diabéticos descompensados que ingresaron por emergencia de un hospital general docente de Chiclayo*.

\begin{tabular}{lccc}
\hline VARIABLE & $\begin{array}{c}\text { Cetoacidosis } \\
\text { diabética }\end{array}$ & $\begin{array}{c}\text { Coma } \\
\text { hiperosmolar }\end{array}$ & p \\
\hline Edad & $55,9 \pm 18,1$ & $53,1 \pm 13,4$ & 0,6110 \\
Tiempo de Diagnóstico & $7,6 \pm 4,2$ & $3,4 \pm 2,4$ & 0,9518 \\
Presión Arterial Media (PAM) & $67,1 \pm 11,7$ & $77,9 \pm 14,7$ & 0,0090 \\
Sodio (mEq/1) & $137,9 \pm 13,8$ & $140,4 \pm 11,1$ & 0,3000 \\
Potasio & $4,9 \pm 2,6$ & $4,6 \pm 1,2$ & 0,5300 \\
Glicemia 0 horas & $397,2 \pm 99,3$ & $646,4 \pm 152,1$ & 0,0000 \\
Urea (mg/dl) & $74,5 \pm 61,7$ & $87,5 \pm 76,1$ & 0,0190 \\
Creatinina (mg/dl) & $2,3 \pm 2,8$ & $2,2 \pm 1,7$ & 0,9300 \\
\hline
\end{tabular}

Las características clínicas, demográficas, ácido base y electrolíticas en cada uno de los grupos de cetoacidosis diabética y coma hiperosmolar solo la acidosis metabólica $(\mathrm{p}=0,000)$ y la alcalosis respiratoria $(\mathrm{p}=0,038)$ demostraron tener diferencia significativa (tabla 4).

Los niveles de presión arterial media, urea y glucosa en sangre, fueron mayores en los pacientes con coma hiperosmolar $(\mathrm{p}<0,05)$ (tabla 5).

No se encontró diferencia entre cetoacidosis diabética y coma hiperosmolar en sexo, índice de masa corporal, debut de la diabetes, en los niveles de la glicemia a la tercera hora, niveles de creatinina, ocupación, tipo de tratamiento de la diabetes, niveles de sodio y potasio al ingreso, frecuencia de falla renal aguda y en la mortalidad (tablas 4 y 5).

\section{DISCUSIÓN}

Un estudio descriptivo en Lima, reportó una baja incidencia de diabetes tipo 1 y la cetoacidosis diabética fue más frecuente en pacientes con diabetes mellitus 2 . Setenta y tres $(74,5 \%)$ correspondieron a cetoacidosis diabética, $9 \quad(9,2 \%)$ a estado hiperglucémico hiperosmolar y $16(16,3 \%)$ a estados mixtos. La hipokalemia fue la complicación más frecuente. En los casos de cetoacidosis, el $42 \%$ de los pacientes tenían la patología de reciente aparición. La mayoría de los episodios de cetoacidosis diabética, fueron graves y se relacionaron con infecciones o falta de cumplimiento en el tratamiento (6).

Las complejidades metabólicas agudas de la diabetes son los episodios de crisis de hiperglucemia (HCE) donde la CD y el EH de hiperglucemia constituyen las emergencias diabéticas más graves, ambas complicaciones son causa de mortalidad. La mortalidad de los sujetos con $\mathrm{HCE}$ es casi 3 veces mayor a los sujetos sin HCE. Después de ajustar la edad, el género y las comorbilidades seleccionadas, el índice de riesgo de mortalidad para los pacientes con HCE fue aún cuatro veces mayor que para los pacientes sin $\mathrm{HCE}$ (7).

La cetoacidosis diabética y la enfermedad hiperosmolar son las dos patologías metabólicas agudas más peligrosas de la diabetes mellitus. En este estudio se evaluaron 52 pacientes con crisis hiperglicémicas; de los cuales el $75 \%$ correspondía a cetoacidosis diabética y el $25 \%$ a síndrome hiperosmolar, no hubo trastornos mixtos. Esto concuerda con los resultados del estudio de Manrique y col. (8), realizado en el Hospital Loayza, quienes encontraron $60 \%$ de cetoacidosis diabética y de ellos, el $4,5 \%$ fueron pacientes con diabetes tipo $1 ; 54,5 \%$ diabetes mellitus $2 ; 33,3 \%$ diabetes no definida y $7,5 \%$ otros tipos (gestacional, corticoides, postpancreatitis y acromegalia).

En nuestro estudio todos los pacientes fueron diabetes mellitus 2; aunque la mayoría de los pacientes con cetoacidosis diabética tienen diabetes autoinmune tipo 1 , los pacientes con diabetes mellitus 2 también están en riesgo durante el estrés catabólico de una enfermedad aguda como traumatismo, cirugía o infección.

En una cohorte poblacional en Taiwan donde se identificaron 7693 sujetos con crisis hiperglicémicas el $30,9 \%$ correspondieron a cetoacidosis diabética y el $69,0 \%$ a enfermedad hiperosmolar (7). Los resultados de este estudio son diferentes a los encontrados en 
nuestro estudio y en el efectuado en el Hospital Loayza. Estas diferencias pueden deberse a que los estudios efectuados en el Perú son series de casos que no representan a la población.

El promedio de la edad de los pacientes con cetoacidosis diabética y enfermedad hiperosmolar de nuestro estudio, fue mayor que el reportado en la literatura $(55,8 \pm 18,1$ vs $53,1 \pm 13,4)$; los pacientes con cetoacidosis diabética, generalmente son jóvenes de mediana edad. En un estudio de 216 casos de cetoacidosis diabética, la edad promedio de los pacientes fue $37,7 \pm 12,4$ años, mucho menor a los datos de nuestra serie (9). La edad promedio de nuestros pacientes con enfermedad hiperglicemica fue 53,1 $\pm 13,4$ años, similar al estudio portugués que encontró un promedio de edad de 54,6 $\pm 9,4$ años (10)

En nuestro estudio se encontraron 14 (26,9\%) pacientes con hiperglicemia con trastorno de la conciencia, hallazgo diferente al encontrado en Portugal donde el $90 \%$ de los pacientes tubo algún grado de alteración de la conciencia y el $28 \%$ estuvo en coma (6); asimismo, encontramos $76,9 \%$ que cursaron con acidosis metabólica, casi el cuádruple del encontrado en el reporte portugués $(22,2 \%)(10)$.

Todos los pacientes de nuestra serie tenían diabetes mellitus 2 y el $15 \%$ eran de debut; a diferencia del estudio de Lima, donde $21 \%$ tenían diabetes mellitus 2 y el $15 \%$ eran de debut (6). Igualmente, no se encontró diferencia según el sexo, aunque los datos de la literatura muestran ligera ventaja en los varones (9).

En nuestra serie, no se encontró diferencia significativa en los valores de sodio y potasio entre pacientes con cetoacidosis diabética y enfermedad hiperosmolar. En la literatura se reporta en los pacientes con cetoacidosis diabética niveles de sodio de $131,6 \pm 6,5$ y de potasio en $5,4 \pm 3,5 \mathrm{mEq} / 1$. Éstos valores de sodio son menores a lo hallado en nuestro estudio $(137,9 \pm 13,8)$; en cambio, los valores de potasio fueron mayores a los de nuestra serie $(4,9 \pm 2,6$ $\mathrm{mEq} / \mathrm{l})(9)$. Estas variaciones pueden tener diferentes explicaciones desde la toma de muestras, técnica de análisis y otros factores que necesitan estudiarse.

En nuestro estudio hubo cuatro fallecidos, tres con cetoacidosis diabética y uno con enfermedad hiperosmolar. La mortalidad después de una crisis hiperglicémica en pacientes de mediana edad, no ancianos, aún no está claro (14). En Taiwan, la mortalidad fue $14,1 \%$ en pacientes con coma hiperglicémico y 4,7\% en pacientes sin coma hiperglicemico; las tasas de mortalidad fueron 3,2 veces más altas en pacientes con coma hiperglicémico que en pacientes sin coma hiperglicémico. Además, la mortalidad en cetoacidosis diabética fue de $17,7 \%$ y $31,9 \%$ en enfermedad hiperosmolar (14). Otros estudios muestran diferentes tasas; por ejemplo, la tasa de mortalidad varía entre $1 \%$ y $9 \%$ en cetoacidosis diabética, de $5 \%$ a $45 \%$ en coma hiperosmolar y $5 \%$ a $25 \%$ en cetoacidosis diabética y coma hiperosmolar mixto $(9,10,11)$. La mortalidad en pacientes con enfermedad hiperosmolar varía entre $10 \%$ y $20 \%$, que es hasta 10 veces mayor que la de cetoacidosis diabética (12).

El coma hiperosmolar fue causa del $27,3 \%$ al $87,6 \%$ de las admisiones a las salas de emergencia y la mortalidad asociada al coma hiperosmolar varía entre $0,2 \%$ y $0,5 \%(20,21,22)$. Ticse $(4)$, reporta una mortalidad de $5,7 \%$ en pacientes con cetoacidosis diabética (4). La tasa de letalidad hasta los 28 días para cetoacidosis y coma hiperosmolar descritas es de $2,7 \%$ y $11,7 \%$, respectivamente (4).

Dentro de las complicaciones agudas, más de la mitad de los pacientes cursaron con injuria renal aguda (IRA), no se encontró diferencia significativa entre cetoacidosis diabética, y enfermedad hiperosmolar $(p=0,74)$. La injuria renal aguda es un trastorno común y corregible en pacientes con una crisis hiperglucémica. En un estudio de Singhal et al. (16), la incidencia de insuficiencia renal aguda en pacientes con crisis diabética (cetoacidosis diabética y enfermedad hiperosmolar) fue $25 \%$ (16).

Se ha descrito que la cetoacidosis diabética, se asocia a varios trastornos del medio interno entre ellos, deshidratación, trastornos del equilibrio ácidobase y de los electrolitos. La injuria renal aguda está relacionada principalmente con la hipovolemia debida a la poliuria osmótica inducida por la glucosa $y$, en ocasiones, a la emesis. Sin embargo, durante años, se utilizaron definiciones no consensuales, lo que dificulta la obtención de una evaluación precisa de la epidemiología de la injuria renal aguda. La disfunción renal aguda sigue siendo poco comunicada en pacientes con injuria renal aguda grave. En un reciente estudio, Wang, evaluó la frecuencia de injuria renal aguda en 94 pacientes con cetoacidosis diabética; de acuerdo con los criterios RIFLE, 50\% presentó injuria renal aguda al ingreso y la mayoría estaba en la clase de riesgo (51\%). A las 12 y 24 horas, el porcentaje de pacientes con KDIGO (AKI) disminuyó a 26\% y 27\%, 
respectivamente. La edad, los niveles de glicemia y el nivel de las proteínas séricas estuvieron asociados mayor riesgo (17). Estos resultados son similares al encontrado en nuestro estudio, aunque no tenemos datos de seguimiento de los pacientes.

No hay datos en la literatura de la frecuencia de injuria renal aguda en pacientes con enfermedad hiperosmolar. Como la deshidratación es más grave en pacientes con enfermedad hiperosmolar que en cetoacidosis diabética, la incidencia de injuria renal aguda en enfermedad hiperosmolar puede ser mayor (17).

Como limitaciones del estudio podemos mencionar que el estudio es una serie de casos, con escasa muestra, transversal y descriptivo. Además, no se realizó análisis de gases y electrolitos en todos los pacientes, por la falta de cobertura del sistema integral de salud.

Finalmente, se puede concluir que la complicación más frecuente fue la Cetoacidosis diabética, y el síntoma más frecuente fue confusión mental; las alteraciones electrolíticas más frecuentes fueron hiponatremia e hipokalemia y la tasa de mortalidad fue baja.

\section{Declaración de financiamiento y de conflictos de intereses:}

El estudio fue financiado por los autores; declaran que no existe conflicto de intereses.

\section{Contribución de autoría:}

RLZ: Contribuyó en la idea de la investigación, diseño del estudio, elaboración y redacción del artículo y aprobación de la versión a ser publicada. GSChP: Toma de muestra y procesamiento de los exámenes de laboratorio, recolección de los datos. CLM: Procesamiento y análisis de los datos, interpretación de los resultados.

\section{Correspondencia:}

Robinson León Zuloeta.

Correo electrónico: robinsonleonzuloeta@gmail.com

\section{REFERENCIAS BIBLIOGRÁFICAS}

1. Seclén SN, Rosas ME, Arias AJ, Huayta E, Medina CA. Prevalence of diabetes and impaired fasting glucose in Perú: report from PERUDIAB, a national urban populatiuon-ased longitudinal study. BMJ Open Diabetes Res Care. 2015; 3(1):86-226. doi: 10.1136/bmjdrc-2015-000110

2. Kitabachi AE; Umpíerrez GE, Miles JM, Fischer JN. Hyperglicemic crisis in adult patients with diabetes. Diabetes Care. 2009; 32(7):1335-43. doi: $10.2337 / \mathrm{dc} 09-9032$

3. Magee MF, Bhatt BA. Management of decompensated diabetes. Diabetic ketoacidosis and hyperglycemic hyperosmolar syndrome. Crit Care Clin. 2001; 17(1):75-106. DOI: 10.1016/s0749-0704(05)70153-6

4. Ticse R, Alán-Peinado A, Baiocchi-Castro L. Características demográficas y epidemiológicas de pacientes con diabetes mellitus tipo 2 hospitalizados por cetoacidosis diabética en un Hospital General de Lima-Perú. Rev Mrd Hered. 2014; 25(1):5-12. DOI: https://doi.org/10.20453/rmh.v25i1.259

5. Pinto M, Villena J, E Villena A. Diabetic Ketoacidosis in Peruvian patients with type 2 Diabetes Mellitus. Endocr Pract. 2008; 14(4):442-6. DOI: 10.4158/ EP.14.4.442

6. Cipriani E. Características demográficas y epidemiológicas de pacientes con diabetes mellitus tipo 2 hospitalizados por cetoacidosis diabética. Rev Med Hered. 2014; 25(3): 183-186. DOI: https://doi. org/10.20453/rmh.v25i3.2000

7. Kao Y, Hsu C-C, Weng S-F, et al. Subsequent mortality after hyperglycemic crisis episode in the non-elderly: a national population-based cohort study. Endocrine. 2016; 51(1):72-82. doi: 10.1007/s12020-015-0669-8

8. Manrique M, Calderón J, Soto A, Calle A, Solis J, Castillo O. Cetoacidosis diabética: una complicación frecuente de la diabetes tipo 2 en hispanoamericanos. Av Diabetol. 2003; 19:141-147.

9. Nyenwe EA, Razavi LN, Kitabachi AE, Khan AN, Wan JY. Acidosis: The Prime Determinant of Depressed Sensorium in Diabetic Ketoacidosis. Diabetes Care. 2010; 33(8):1837-9. doi: 10.2337/ dc10-0102

10. Vitor M, Bastos M, Martins T, Lemos M, Ruas A. Hiperosmolaridad diabética. Análisis retrospectivo de 60 casos. Acta Med Port. 2003; 16:13-19.

11. Chou W, Chung MH, Wang HY, et al. Clinical characteristics of hyperglycemic crises in patients without a history of diabetes. J Diabetes Invest. 2014; 5: 657-662.

12. Pasquel FJ, Umpierrez GE. Hyperosmolar hyperglycemic state: a historic review of the clinical presentation, diagnosis, and treatment. Diabetes Care. 2014; 37(11):3124-31.

13. Trachtenbarg DE. Diabetic ketoacidosis. Am Fam Physician. 2005; 71(9): 1705-1714.

14. Yeo K-F, Yang Y-S, Chen K-S, Peng C-H, Huang C-N. Simultaneous presentation of thyrotoxicosis and diabetic ketoacidosis resulted in sudden cardiac 
arrest. Endocr J. 2007; 54(6):991-3.

15. Wang J, Geiss LS, Williams DE, Gregg EW. Trends in Emergency Department visit rates for hypoglycemia and hyperglycemic crisis among adults with diabetes, United States, 2006-2011. PLoS One. 2015; 10(8): e0134917. doi: 10.1371/journal.pone.0134917

16. Singhal PC, Abramovici M, Ayer S, Desroches L. Determinants of rhabdomyolysis in the diabetic state. Am J Nephrol. 1991; 11(6):447-50. DOI: $10.1159 / 000168357$
17. Orban J-C, Maizière E-M, Ghaddab A, Van Obberghen $\mathrm{E}$, Ichai $\mathrm{C}$. Incidence and characteristics of acute kidney injury in severe diabetic ketoacidosis. PLoS One. 2014; 9(10):e110925. doi: 10.1371/ journal.pone.0110925

18. Wang LM, Tsai ST, Ho LT, Hu SC, Lee CH. Rhabdomyolysis in diabetic emergencies. Diabetes Res Clin Pract. 1994; 26(3):209-14.

Recibido: 03/06/2019

Aceptado: 31/03/2020 